\title{
Magnetically modulated optical nanoprobes
}

\author{
Jeffrey N. Anker and Raoul Kopelman ${ }^{\mathrm{a})}$ \\ The University of Michigan Chemistry Department, Ann Arbor, Michigan 48109-1055
}

(Received 23 July 2002; accepted 17 December 2002)

\begin{abstract}
We have developed magnetically modulated optical nanoprobes (MagMOONs) to magnetically modulate the signal from fluorescent probes and thus separate it from autofluorescence, electronic offsets, and other background signals. These micro- and nanosized particles emit fluorescence signals, indicating chemical concentrations, and blink in response to rotating magnetic fields. Demodulating the signal dramatically enhances the probe's signal to background ratio. The probes and methods promise to improve immunoassays, intracellular chemical sensing, and fundamental biochemical research. (C) 2003 American Institute of Physics. [DOI: 10.1063/1.1544435]
\end{abstract}

Fluorescence is the most sensitive method available for molecular detection and chemical imaging. It is even used for real time imaging of single molecules at ambient conditions with high spatial and spectral resolution. ${ }^{1,2}$ Fluorescent dyes are commonly used to study intracellular chemical concentration changes, ${ }^{3,4}$ measure immunochemical concentrations in fluids, ${ }^{5}$ tag molecules on the surface of cells and tissues, ${ }^{6}$ and research protein folding. ${ }^{7,8}$ They are critical to understanding how cells function, for rapid drug discovery, and for detecting minute quantities of pathogens and DNA. Each fluorescent dye typically emits tens of thousands to hundreds of thousands of photons before photobleaching. Intense, low-shot-noise signals can be accurately detected with highly linear optical sensors such as photodiodes, CCDs, and photomultiplier tubes. Nevertheless, background fluorescence from sample and instrument optics can drown out probe fluorescence which makes detecting and distinguishing low levels of probe fluorescence and small changes in probe fluorescence a challenging endeavor. Rejecting background fluorescence and increasing signal-to-noise $(\mathrm{S} / \mathrm{N})$ ratios by orders of magnitude would thus lead to important advances in molecular and biomedical sciences.

Blinking or flashing lights are easily distinguished from lights that remain on constantly or that slowly change their intensity. It is an established scientific practice to modulate signals in order to increase $\mathrm{S} / \mathrm{N}$ ratios. We have developed magnetically modulated optical nanoprobes (MagMOONs) that blink in response to rotating magnetic fields. By separating the blinking probe signal from the unmodulated background, we can simply and sensitively detect low chemical concentrations even in the presence of autofluorescence and other backgrounds. Thus MagMOONs can extend the range of dyes that may be used for sensing and also extend the range of conditions under which experiments will work. In contrast to chemiluminescence, fluorescence from MagMOONs can be hundreds of thousands of times more intense. In contrast to physical or chemical probe separation techniques, the modulation of optical properties reduces sample preparation time as well as instrument complexity. Importantly, the MagMOON rotation rate is independent of

${ }^{a)}$ Electronic mail: kopelman@umich.edu particle size, assuming constant viscosity and magnetic susceptibility. ${ }^{9}$

Fluorescent polystyrene microspheres $4.4 \mu \mathrm{m}$ in diameter containing ferromagnetic material (Spherotech, Libertyville, IL) are deposited on a microscope slide, and one hemisphere of the particles is coated with either vapordeposited aluminum or sputter-coated gold. The metal layer prevents excitation light from entering as well as fluorescence from leaving the coated side of the particle. The metal layer should ideally be thicker than the skin depth of the excitation or emission light, although thinner layers will still allow modulation. We used aluminum layers $100 \mathrm{~nm}$ thick (skin depth on the order of $20 \mathrm{~nm}$ ). Quenching of the dye molecules by the metal is not a problem, since most of the dyes are not in immediate proximity $(<10 \mathrm{~nm})$ to the metal. The microspheres are magnetized so that their north side is uncoated. The particles are then removed from the slide with a paint brush and suspended in solution by sonication. When in solution, the particles orient in an external magnetic field. By rotating the field, the particles are made to rotate, and appear to blink synchronously as their light emitting sides come in and out of view [Fig. 1(a)]. ${ }^{10}$ Since only the probes rotate, any constant background fluorescence can be separated from the probe signal.

Figure 1(b) illustrates a setup to magnetically modulate MagMOONs and separate their blinking signal from unmodulated backgrounds. A program written in LABVIEW (National Instruments, Austin, TX) controls a stepper motor interfaced to a computer parallel port. The motor rotates a magnet clockwise and anticlockwise $180^{\circ}$ in order to orient the particle ON and OFF. Spectra are acquired and saved after every rotation. The probe spectrum is calculated as the average ON minus OFF spectrum. The sample is viewed with an Olympus X70 epifluorescent microscope.

In this letter, we focus on metal-capped MagMOONs. Aspherical magnetic particles give another type of MagMOON. ${ }^{11}$ The aspherical particles orient in magnetic fields due to magnetic shape anisotropy and emit differently from their side than from their ends due to self-absorption within the particles (analogous to Venetian blinds). The two MagMOON types can be distinguished for increased signal multiplexing because only $90^{\circ}$ separates the aspherical MagMOONs' ON and OFF states, as opposed to $180^{\circ}$ for metal- 
(a)

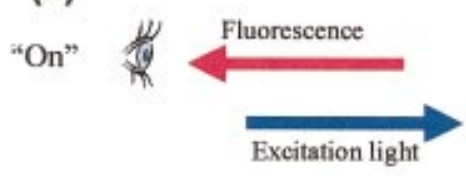

Magnetic Field

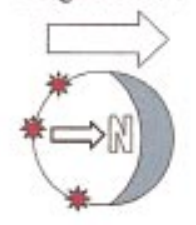

"Off"
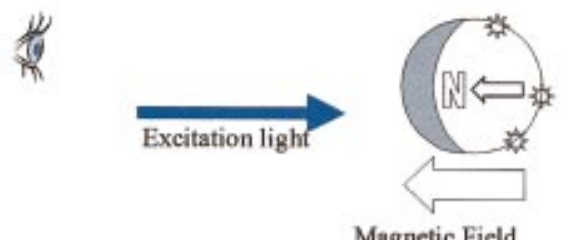

Magnetic Field

\section{(b)}

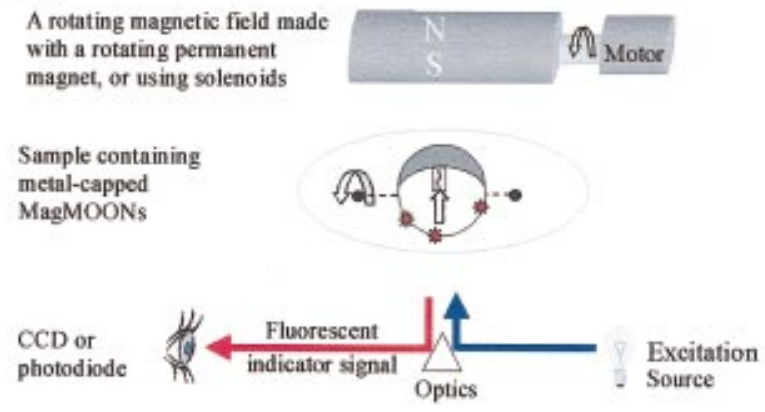

FIG. 1. (Color) (a) An external magnetic field orients the aluminum-capped MagMOON, causing its fluorescent excitation and observed emission to blink on and off as it rotates. (b) A setup to magnetically modulate MagMOONs and separate their blinking signal from the unmodulated background.

capped MagMOONs Probes that respond to modulated magnetic field gradients instead of field orientations give yet another distinguishable type of MagMOON. We have also made 300-nm and 1- $\mu \mathrm{m}$ "Brownian MOONs," in which random thermal motion causes metal-capped particles to tumble and blink erratically.

To demonstrate the principle of MagMOON immunoassays (Fig. 2), gold-capped streptavidin coated MagMOONs were generated by sputter coating gold onto one hemisphere of streptavidin coated permanent magnetic microspheres (Spherotech). These MagMOONs were then immersed in solutions with a fixed concentration of Oregon-Green-labeled biotin (OG) and varying concentrations of phycoerythrinlabeled biotin (PE). The biotin-streptavidin bond, a strong and highly specific biological bond used as the basis for many immunoassays, attached the fluorophores to the MagMOON [Fig. 2(a)]. Although in a traditional immunoassay excess dye would be washed away, in this experiment, no washing step was performed since fluorescence from the excess dye is not modulated and can be subtracted off. A 20- $\mu$ l drop of MagMOON and dye solution was placed on a glass microscope slide that was silanized to keep the drop compact. Some of the particles stuck to the glass surface, so the $4 \times$ objective was focused on particles floating in solution above the glass. By rotating the MagMOONs with the computer controlled magnet, we were able to separate the MagMOON fluorescence from background fluorescence due to instrument optics, contamination from other microspheres (a)
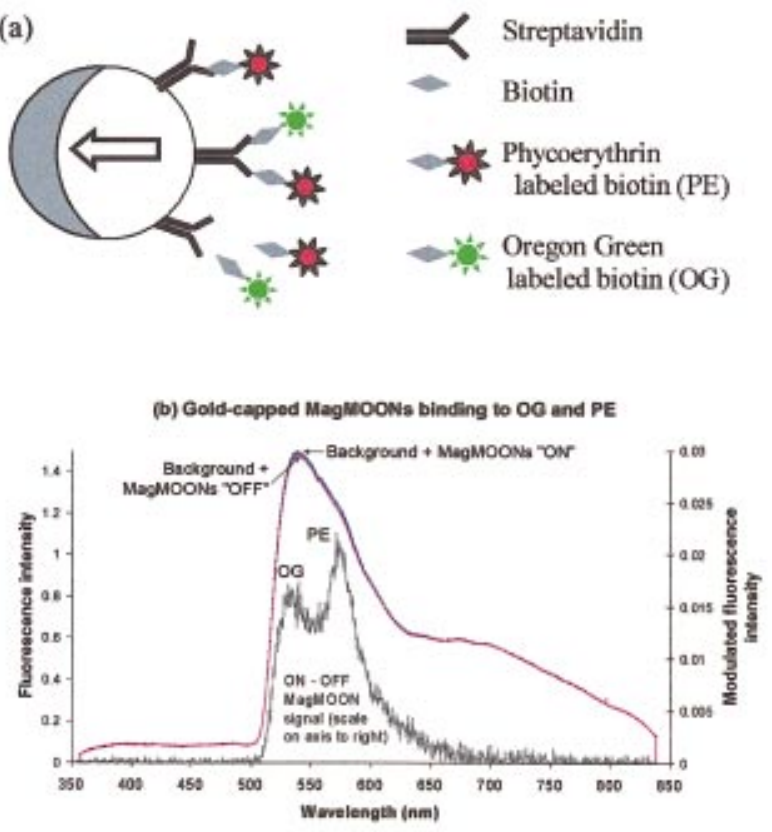

(c) MagMOON Immunoassay measures relative concentration of OG to PE in solution

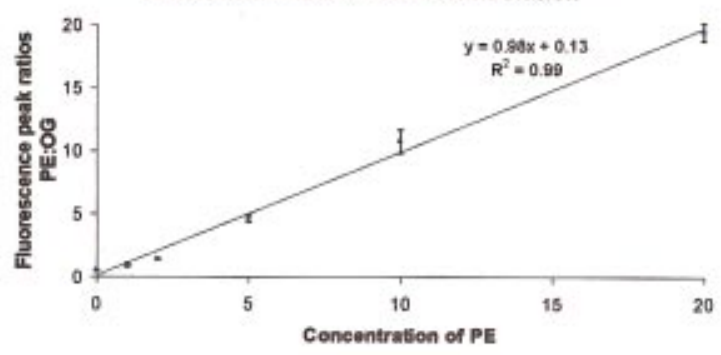

FIG. 2. (Color) Gold-capped, streptavidin-coated MagMOONs detect concentrations of OG and PE even in the presence of background. (a) Assay schematic. (b) MagMOON spectrum is separated from background spectrum. (c) MagMOON immunoassay detects relative concentration of fluorescently labeled biotin. The standard error was calculated from the variation in fluorescence intensity at wavelengths close to the spectral peaks.

added to the solution, and free excess biotin-labeled dyes [Fig. 2(b)]. Experiments were performed with six different $\mathrm{PE}$ concentrations. As expected, the ratio of PE to OG spectral peaks from the MagMOONs increased linearly with concentration of PE in solution, unaffected by a 1000 times stronger background fluorescence [Fig. 2(c)].

Magnetic modulation of optical signals is thus a general technique that rejects background and increases $\mathrm{S} / \mathrm{N}$ ratios for any dye or optical label that can be attached to or em-

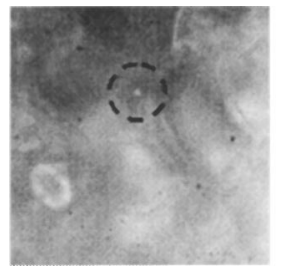

(a) MagMOON "on"
+ leaf

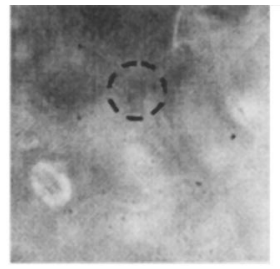

(b) MagMOON "off" + leaf

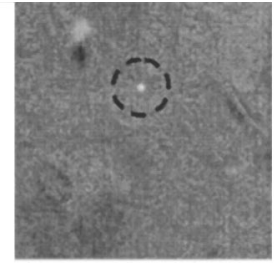

(c) Image subtraction removes leaf background
FIG. 3. A metal-capped MagMOON rotating below a highly fluorescent leaf section. (a) ON. (b) OFF (c) image subtraction, ON minus OFF, removes the background leaf fluorescence. 


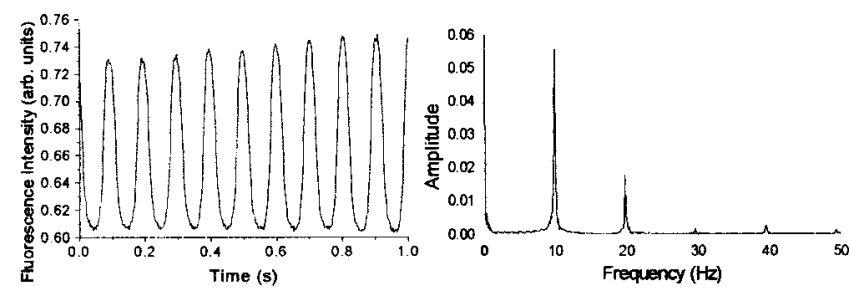

FIG. 4. Magnetic modulation of aluminum-capped MagMOONs. (i) Fluorescence intensity change with respect to time. (ii) Fourier transform of (i).

bedded within MagMOONs. In addition to improved immunoassays, MagMOONs promise to improve intracellular measurements in which autofluorescence has severely limited the range of dyes and samples that can be detected with reasonable $\mathrm{S} / \mathrm{N}$ ratios. Our recently developed probe encapsulated by biologically localized embedding (PEBBLE) nanosensors measure concentrations of ions and small molecules within a single cell, rapidly, sensitively, with high spatial resolution, and without interference from cellular proteins. ${ }^{3}$ Combining them with MagMOONs will allow highly sensitive detection of intracellular analytes using a broad range of dyes. Such measurements lead to a better understanding of how cells function and how cells are effected by drugs, toxins and pathogens. Magnetic particles have been moved and rotated within cells for the past fifty years, principally to measure viscosity. ${ }^{10,12,13}$ However, none of those particles were chemical sensors.

Blinking MagMOONs may also be spatially located by subtracting "on" minus "off" images. Locating MagMOONs and reading their fluorescent signals promises to enable high-sensitivity measurement of chemical concentrations in cells and tissues, high contrast molecular tags and contrast agents, and the ability to perform thousands of bioassays simultaneously in a single fluid sample by first locating the MagMOON and reading its signal, and then identifying it using an optical encoding scheme. ${ }^{14}$ To demonstrate particle localization and background subtraction in images, we sliced thin sections of an ivy leaf and placed them on a microscope slide with a few drops of dilute, aluminumcapped MagMOON solution, wetting the slide below the leaf. A magnet was rotated continuously with a motor drive. Although the leaf fluoresced brightly, and MagMOONs were often initially out of focus, blinking MagMOONs were easily distinguished from leaf fluorescence. Figure 3 shows images of an aluminum-capped MagMOON floating below a brightly fluorescing leaf section. Figure 3(a) shows the MagMOON magnetically oriented ON; Fig. 3(b) shows the MagMOON magnetically oriented OFF: Fig. 3(c) shows ON minus OFF (which removes the background leaf fluorescence). Multiple image subtractions would further increase contrast.

Portable MagMOON immunoassays and chemical sensors may be based on simple devices containing rotating per- manent magnets or solenoids, photodiodes to measure light intensity at specific wavelength regions, and electronic filters to separate MagMOON fluorescence from background. To demonstrate the principle, a magnet was rotated continuously at $10 \mathrm{~Hz}$ above a drop of water containing aluminum-capped MagMOONs. Fluorescence intensity from instrument autofluorescence and rotating (blinking) MagMOONs was measured using a photomultiplier tube (Fig. 4). Although the blinking signal was only a tenth of the constant background signal, the signal at $10 \mathrm{~Hz}$ was 400 times the background signal at $15 \mathrm{~Hz}$, for a total factor of 4000 signal over background increase. Harmonics at 20,30, 40, and $50 \mathrm{~Hz}$ are also present in the Fourier transform. Only $10 \mathrm{~s}$ of data were analyzed for this graph. With more data the lines would become sharper and $\mathrm{S} / \mathrm{N}$ ratio would increase.

In conclusion, we have developed a general technique to magnetically modulate an optical probe signal and separate it from background fluorescence. Metal-capped MagMOON signals have been separated from optical and electronic background by subtracting (ON minus OFF) spectra and images, in addition to continuously rotating probes and taking the Fourier transform of the time series. MagMOONs promise to enhance the $\mathrm{S} / \mathrm{N}$ ratio of a variety of biochemical applications, including immunoassays, intracellular chemical sensors, cellular labels or tags, and protein folding studies.

We thank Dr. E. Monson for his technical assistance and Dr. M. Philbert for his biological insights. We also gratefully acknowledge support from NSF Grant No. DMR 9900434 and NIH/NCI Contract No. N01-CO-07013.

${ }^{1}$ T. A. Byassee, W. C. W. Chan, and S. M. Nie, Anal. Chem. 72, 5606 (2000).

${ }^{2}$ R. Kopelman and W. H. Tan, Science 262, 1382 (1993).

${ }^{3}$ E. Monson, M. Brasuel, and M. A. Philbert, Biomedical Photonic Handbook, edited by T. Vo-Dinh (in press).

${ }^{4}$ R. P. Haugland, Handbook of Fluorescent Probes and Research Chemicals, sixth ed. (Molecular Probes, Inc., Eugene OR., 1996).

${ }^{5}$ K. R. Rogers, Mol. Biotechnol. 14, 109 (2000).

${ }^{6}$ Z. Darzynkiewicz, E. Bedner, X. Li, W. Gorczyca, and M. R. Melamed, Exp. Cell Res. 249, 1 (1999).

${ }^{7}$ V. Subramaniam, A. Gafni, and D. G. Steel, IEEE J. Sel. Top. Quantum Electron. 2, 1107 (1996).

${ }^{8}$ X. W. Zhuang, H. Kim, M. J. B. Pereira, H. P. Babcock, N. G. Walter, and S. Chu, Science 296, 1473 (2002).

${ }^{9}$ P. A. Valberg and J. P. Butler, Biophys. J. 52, 537 (1987).

${ }^{10}$ See EPAPS Document No. E-APPLAB-82-028307 for two videos of MagMOONs' synchronously rotating and blinking. A direct link to this document may be found in the online article's HTML reference section. The document may also be reached via the EPAPS homepage (http:// www.aip.org/pubservs/epaps.html) or from ftp.aip.org in the directory /epaps/. See the EPAPS homepage for more information.

${ }^{11}$ J. N. Anker, C. Behrend, and R. Kopelman, J. Appl. Phys. (in press).

${ }^{12}$ F. H. C. Crick and A. F. W. Hughes, Exp. Cell. Res. 1, 37 (1950).

${ }^{13}$ M. Keller, J. Schilling, and E. Sackmann, Rev. Sci. Instrum. 72, 3626 (2001).

${ }^{14}$ K. Braeckmans, S. C. De Smedt, M. Leblans, R. Pauwels, and J. Demeester, Nature Reviews Drug Discovery 1, 447 (2002). 